\title{
La théorie des signes locaux de Hermann Lotze et la controverse empirisme-nativisme au $\mathrm{XIX}^{\mathrm{e}}$ siècle
}

\author{
Denis FISETTE \\ Université du Québec à Montréal, Montréal (Canada)
}

La théorie des signes locaux de Hermann Lotze représente sa contribution principale au problème de la perception de l'espace. Bien que Lotze n'ait jamais élaboré une théorie en bonne et due forme des signes locaux et que sa conception a évolué entre son hypothèse initiale en 1846 jusque dans ses derniers travaux en $1881^{1}$, sa conception des signes locaux a exercé énormément d'influence sur les principaux artisans de la nouvelle psychologie au XIX ${ }^{\mathrm{e}}$ siècle, et notamment sur Hermann von Helmholtz, Wilhelm Wundt, Ernst Mach et son collègue à Prague le physiologiste autrichien Ewald Hering (cf. Ribot, 1879 ; Ackerknecht, 1904 ; Woodward, 1978). Elle a servi de fil conducteur à son étudiant Carl Stumpf dans un ouvrage dédié à Lotze et publié en 1873 sous le titre Sur l'origine psychologique de la représentation de l'espace, dans lequel il commente la fameuse controverse opposant notamment Helmholtz et Hering sur la perception de l'espace. Cet ouvrage de Stumpf présente un intérêt particulier pour la présente étude non seulement parce qu'il représente un exposé remarquable de la théorie des signes locaux de Lotze et de la fameuse controverse opposant le nativisme à l'empirisme, mais aussi en raison de l'influence qu'il a exercée sur la phénoménologie naissante.

Rappelons d'abord que Stumpf doit une partie de sa formation à Franz Brentano avec qui il entreprit ses études en philosophie à Würzburg en 1867. Mais puisque Brentano n'était pas encore habilité à assurer la direction de ses étudiants, il recommanda à Stumpf et plus tard à Anton Marty de se rendre à Göttingen afin de poursuivre leur formation auprès de Lotze. C'est donc sous la direction de Lotze que Stumpf a soutenu

Lotze élabore différents aspects de sa théorie des signes locaux dans plusieurs ouvrages dont les plus importants sont les suivants : (Lotze, $1846: 172-190 ; 1852: 325-352$; 1856 : 330-347 ; $1879: 543-573 ; 1881: 26-38 ; 1873: 315-324 ; 1877: 345-365)$. 
avec succès sa dissertation sur Platon en 1868 et sa thèse d'habilitation sur les axiomes mathématiques en 1870. Il devient alors, à l'âge de 22 ans, Privatdozent à l'Université de Göttingen et collègue de Lotze jusqu'à son départ en 1873 alors qu'il hérite de la chaire de Brentano à Würzburg. Cette date marque le début d'une longue carrière universitaire qui a duré presque cinquante années dans les universités prestigieuses telles que Prague, Halle, Munich et surtout Berlin où il s'est imposé tant sur le plan institutionnel, philosophique que scientifique (cf. Fisette, 2008; 2015).

Dans son discours inaugural à l'université de Berlin en 1895, Stumpf compare l'influence qu'il a reçue de Brentano et de Lotze durant ses années d'étude à Würzburg et Göttingen à une époque où les grands systèmes spéculatifs étaient en déclin tandis que l'orientation empirique était de plus en plus valorisée :

Franz Brentano m'a indiqué cette voie et m'a aussi fourni, grâce à sa connaissance fine et érudite d'Aristote, des suggestions décisives et détaillées de même que les germes de plusieurs idées ; alors que l'influence plus tardive de Lotze m'a transmis en particulier l'intérêt pour les objets psychologiques et l'habitude des fondations élargies (Stumpf, 1895 : 735).

Bien que ses études à Würzburg auprès de Brentano aient été relativement courtes comparativement à la durée de ses études et de son séjour à Göttingen auprès de Lotze, le germe de la plupart de ses idées philosophiques est à chercher dans l'enseignement qu'il a reçu de Brentano à Würzburg. Ce qui ne veut pas dire que Lotze n'était pas également une source d'inspiration importante pour Stumpf, comme le confirme le mémoire publié par Stumpf dans les Kant-Studien à l'occasion du centenaire de la naissance de Lotze. Il explique que si Brentano a pu lui recommander de poursuivre ses études auprès de Lotze, c'est entre autres choses en raison de l'approche scientifique qu'il préconisait en philosophie :

Parmi les professeurs allemands de philosophie, aucun ne pouvait être mis même approximativement au même rang que lui si, comme Brentano luimême, on considérait la méthode de la pensée dans les sciences de la nature comme exemplaire pour la philosophie et un contact étroit avec les sciences de la nature comme la condition d'une pratique efficace de la philosophie (Stumpf, $1917: 2$ ).

Dans « La renaissance de la philosophie », la philosophie de Lotze se présente comme l'antithèse des grands systèmes idéalistes et Stumpf lui assigne une place centrale dans ce segment de l'histoire de la philosophie. Il y distingue en effet deux voies ou orientations prises par la philosophie au XIX ${ }^{\mathrm{e}}$ siècle, la première étant associée, comme il se doit, à l'idéalisme kantien, tandis que la seconde, qu'il appelle Erfahrungsphilosophie, commence avec Lotze qui « a pénétré, avec une acuité extraordinaire, les 
problèmes philosophiques dans toute leur profondeur » (Stumpf, 1907 : 165). Stumpf soutient que seule la voie ouverte par Lotze peut conduire à une véritable renaissance de la philosophie.

C'est durant son séjour à Göttingen que Stumpf entreprend la rédaction de son Raumbuch. Dans son autobiographie, Stumpf raconte qu'il avait d'abord entrepris en 1870 de vastes recherches sur l'histoire de la notion de substance, projet qu'il abandonna en 1872 au profit de son étude sur la perception de l'espace :

Le premier travail important que j'entrepris fut l'histoire critique du concept de substance, qui me donna d'épouvantables maux de tête et que j'abandonnai pour m'attaquer, à Pâques de 1872, au thème psychologique de l'origine de la représentation de l'espace. Je croyais (et crois encore) que la relation entre la couleur et l'étendue était à concevoir comme un exemple frappant ou un analogon de la relation que la métaphysique admettait entre les propriétés d'une substance. D'où la connexion entre le thème nouveau et mon travail ancien (Stumpf, $1924: 8$ ).

Suivant cette indication, le lien entre le thème psychologique, c'està-dire l'origine de la représentation de l'espace, et le thème ontologique dans le Raumbuch est à chercher dans la théorie des touts et des parties sur laquelle est fondée la position de Stumpf dont il sera question plus loin. Dans son ouvrage posthume Erkenntnislehre, Stumpf explique que la notion de partie psychologique s'est imposée à lui dans ses recherches sur l'histoire de la notion de substance et qu'elle est aussi redevable à Brentano comme le confirme également une note de son ouvrage posthume (1939: 183 ; et 24) dans laquelle Stumpf nous apprend en outre qu'il a adopté cette position au moment où la première partie de l'ouvrage était déjà chez l'imprimeur et que c'est sous l'influence de Brentano qu'il aurait alors abandonné sa position initiale, qui était proche de celle de Lotze, voire même de l'empirisme qu'il critique dans la deuxième partie de l'ouvrage. Il n'en reste pas moins que les connaissances acquises par Stumpf dans le domaine de la psychologie scientifique - il n'a jamais suivi les leçons de Brentano sur la psychologie - et l'intérêt qu'il a pris aux débats sur la perception de l'espace sont redevables de ses fréquentations de Lotze durant son séjour à Göttingen.

Ces remarques biographiques sont utiles pour bien comprendre la position que défend Stumpf face au débat opposant le nativisme et l'empirisme sur le problème de l'origine des représentations de l'espace. Rappelons d'abord que cette controverse porte en définitive sur la formation de l'espace (de l'ordre spatial), et plus précisément sur la relation entre l'étendue et les qualités visuelles et tactiles, de même que sur la localisation de ces qualités dans l'espace. La prémisse commune aux empiristes et aux nativistes est que nos sensations diffèrent 
qualitativement les unes des autres suivant leur lieu d'origine ou suivant le lieu où elles sont produites, et la fonction du signe local consiste précisément à désigner son lieu d'origine ou sa cause. Mais les deux partis ne s'entendent pas sur le type de relation liant l'espace et la qualité ou le signe local avec son lieu d'origine, et sur la nature même des qualités sensorielles et des signes locaux. Pour les nativistes, cette relation entre qualité et étendue est intrinsèque aux contenus sensoriels tandis que les empiristes estiment qu'elle est extrinsèque et de nature judicative ou associative. Kant, Lotze et les empiristes soutiennent que les données immédiates de la conscience sont des sensations brutes, une mosaïque de sensations, tandis que les nativistes y voient des phénomènes organisés et structurés selon des lois structurelles qui leur sont propres. Un empiriste comme Helmholtz, par exemple, conçoit les signes locaux comme de simples marques distinctives dont la signification s'épuise entièrement dans l'interprétation qui l'anime. En revanche, les nativistes estiment que les signes locaux renvoient immédiatement à des différences locales en ce sens que le contenu de cette sensation locale nous fournit immédiatement le lieu de son origine. Les nativistes admettent donc avec Kant qu'il n'y a pas de qualité sans étendue, mais ils reconnaissent avec les empiristes qu'il n'y a pas d'espace sans qualité ou matériau sensible. Ils admettent en outre que l'espace procède de l'intuition, mais ils estiment que la relation entre qualité et espace n'est pas de nature judicative ou associative, mais qu'elle est inhérente aux contenus sensoriels ou aux phénomènes eux-mêmes.

Cette courte description donne une mince idée de l'ampleur de cette controverse, de son importance historique et de sa portée sur l'histoire de la philosophie en Allemagne durant la deuxième moitié du XIX ${ }^{\mathrm{e}}$ siècle. Elle est en bonne partie responsable de la grande division au sein de la nouvelle psychologie entre le courant empiriste, pour reprendre l'expression plus ou moins adéquate de Helmholtz - il vaudrait mieux parler ici d'une forme d'intellectualisme ou de mentalisme comme nous le verrons plus loin ; le courant opposé est issu des travaux de Hering en physiologie, et il passe par l'école descriptiviste de Kirchhoff en physique dont le représentant le plus connu est Ernst Mach, par l'école de Brentano en psychologie, et il aboutit comme on le sait à la psychologie de la forme. Or, c'est justement à ce dernier courant qu'appartiennent les différentes versions de la phénoménologie comprise dans un sens général comme doctrine des éléments ou phénomènes sensibles. Cette phénoménologie a été comprise à l'origine comme une science neutre, comme une espèce de propédeutique à la science en ce sens que son champ d'étude était considéré comme un domaine commun à des sciences comme la physique, la physiologie et la psychologie, par exemple, et que son travail représentait un passage obligé des autres sciences. 
Vu sous cet angle, un des enjeux importants de cette controverse concerne peut-être moins l'opposition entre l'empirisme et le nativisme, que celui opposant une forme de constructivisme, commune aux kantiens et aux empiristes et suivant laquelle le donné est un construit (au moyen des catégories de l'entendement ou des lois de l'association) et une approche phénoménologique qui prend sa mesure dans la description du perçu. Cette approche a l'immense avantage de rendre possible une première délimitation du champ d'action de la phénoménologie avant Husserl, et donc d'une phénoménologie comprise dans un sens large.

\section{La théorie des signes locaux de Lotze}

Dans sa version initiale, l'hypothèse de signes locaux répondait à la question de savoir comment les qualités de couleur sont localisées de manière déterminée dans l'espace, pourquoi un seul et même rouge apparaît tantôt à tel endroit, tantôt à tel autre, et que, en règle générale, les couleurs sont réparties de manière structurée dans le champ visuel. Le problème de la localisation est ainsi de savoir comment des qualités non spatiales, associées avec des points particuliers sur la rétine, permettent à l'œil de distinguer entre des sensations identiques, le même rouge, issu de la stimulation de différents points sur la rétine. Car ces indices permettent bien de distinguer qualitativement deux sensations $a$ et $b$, mais pour établir une relation spatiale entre ces deux sensations, il doit y avoir dans notre organisation sensible des motifs ou indications qui, suivant l'hypothèse de Lotze, incitent l'âme à « reconstruire la relation spatiale entre les objets $a$ et $b$, c'est-à-dire de représenter les objets $a$ et $b$ l'un à côté de l'autre » (cf. Lotze, $1852: 327$ sq.).

Ainsi formulé, le problème de la localisation porte sur les conditions qui rendent possible notre représentation des objets dans le même ordre spatial qu'ils occupent à l'extérieur de nous. L'hypothèse des signes locaux repose sur l'idée que les différences spatiales et les relations entre les impressions sur la rétine doivent compenser pour les relations simplement intensives et non spatiales correspondantes entre les sensations, et c'est par un procédé psychologique que l'âme transforme à nouveau ces données intensives en données extensives, c'est-à-dire l'arrangement de ces sensations dans l'espace. Cet arrangement spatial est en fait une « reconstruction » de l'espace, une espèce de représentation mentale qui, sur la base des indications que lui fournissent les signes locaux, rapporte ces données intensives aux objets extérieurs ou à des parties du corps. Ainsi, pour expliquer l'ordre spatial des qualités de couleur dans le sens visuel, qui ne contiennent en soi aucune étendue ou aucun ordre spatial, nous devons présupposer, selon l'hypothèse de Lotze, qu'ils véhiculent 
certains indices qui nous permettent de déterminer l'ordre initial. Ce sont ces indices que Lotze appelle des signes locaux.

Prenons une configuration formée de trois objets $A, B, C$, qui se présente comme la figure sensible d'un triangle, ces trois points correspondant aux trois sommets d'un triangle, $A$ se situant à gauche de $B$ et de $C$, et audessus de $A$ et de $B$. Ces trois éléments sont perçus grâce à l'action qu'ils exercent sur les places correspondantes de la rétine, soit les stimulations $p, q$, et $r$. Mais ces stimulations n'expliquent pas à elles seules comment nous déterminons leur position, car les relations spatiales entre $A, B$ et $C$ disparaissent au moment où les données extensives se transforment en données intensives $p, q, r$, en supposant, comme le fait Lotze, que les données intensives n'ont pas d'étendue et ne présentent donc aucune propriété spatiale.

Lotze (1856 : 334-5) compare le passage des données extensives aux données intensives au déménagement d'une bibliothèque d'un lieu à un autre. Il existe en effet plusieurs façons d'ordonner et de classer des éléments dans l'espace, et dans le cas de la localisation, le problème est de savoir comment nous pouvons reconstituer l'ordre initial des ouvrages après le déménagement, c'est-à-dire comment nous pouvons assigner une place à chacun des ouvrages, en faisant abstraction de la question de savoir pourquoi cette localisation doit se faire suivant l'ordre initial. Car puisque l'ordre initial disparait lorsqu'on place les ouvrages dans des cartons, nous avons besoin d'indications pour le reconstituer dans un autre lieu, nous avons besoin d'étiquettes ou de labels, par exemple, qui auraient été accolés à chacun des ouvrages afin de marquer leur position initiale les uns par rapport aux autres. De manière analogue, pour expliquer l'ordre spatial des qualités de couleur dans le sens visuel, qui ne contiennent en soi aucune étendue ou aucun ordre spatial, comme les livres durant le déménagement, nous devons présupposer qu'ils véhiculent certains indices qui nous permettent de déterminer un ordre quelconque, et ce sont ces indices que Lotze appelle des signes locaux. Mais ces indices ne suffisent pas à eux seuls pour déterminer l'ordre initial puisque les ouvrages de la bibliothèque pourraient aussi bien être ordonnés suivant la grosseur, la couleur de leur couverture, suivant la date de leur parution, leur contenu, l'ordre alphabétique du nom de l'auteur, etc. Qu'est-ce qui rend possible la distinction entre telle classification et telle autre ? Dans ces conditions, ne doit-on pas admettre, demande Stumpf, un contenu positif de représentation qui justifierait telle ou telle classification ou plus précisément tel ordonnancement spatial par rapport à tel autre et qui permettrait de distinguer cette forme d'ordonnancement de la forme temporelle, par exemple ? Autrement dit, comment pourrionsnous ordonner spatialement les sensations pures si, comme le pense Kant, 
elles ne possèdent aucun trait spatial ? Stumpf soutient au contraire qu'il n'y a pas d'ordre ou de relation sans un contenu positif qui les fonde.

Pour expliquer comment, en prenant comme point de départ les seules qualités sensibles, qui ne contiennent en soi aucune étendue ou aucun ordre spatial, comme les livres durant le déménagement, nous pouvons déterminer l'ordre initial, nous devons présupposer dans l'âme un dispositif quelconque qui explique la coordination spatiale des sensations visuelles et tactiles. Autrement dit, le problème de l'espace pour Lotze en est un de reconstruction des données extensives à partir des données intensives, et il consiste à « faire renaître tout de nouveau l'image étendue qui s'est effacée, et par conséquent être en état d'assigner à chaque impression la situation relative qu'il lui faut occuper, dans cette image, à côté des autres ». Pour ce faire, nous devons présupposer que ces sensations visuelles et tactiles véhiculent des indices et nous fournissent un guide quelconque "d'après les indications duquel [on] puisse trouver, pour chacune, la place convenable afin que l'image-idée naissante ressemble à l'image-espace évanouie » (Lotze, 1879 : 569). Ces indices sont justement des signes ou des indices qu'il appelle « locaux» parce que les impressions qui les provoquent sont porteuses de leur lieu d'origine dans l'excitation qui en est la cause, de la même manière que la fumée peut servir d'indice à sa cause et renvoyer ainsi au feu.

La métaphore de la lentille utilisée par Lotze dans sa Métaphysique traduit parfaitement ce procédé de reconstruction :

[E]lle condenserait en un seul point indivisible tous les rayons réfléchis par une surface éclairée ; en ce point, il n'y aurait plus à distinguer la position relative des rayons qui s'y trouveraient concentrés et n'y formeraient qu'une clarté unique ; mais au-delà de ce point, les rayons reprendraient leur divergence et dessineraient sur un plan opposé la copie fidèle de la surface donnée. Nous comparons aux rayons qui se dirigent vers la lentille les mouvements nerveux qui tendent à agir sur l'âme ; au point de concentration correspond l'unité de la conscience ; seule la reconstruction dans l'âme des relations d'espace d'abord anéanties diffèrent sensiblement de la divergence des rayons qui n'est que la simple continuation d'un mouvement antérieur (Lotze, 1877 : 346).

La figure suivante, que je tire de l'ouvrage de Pastore (1971), illustre clairement cette métaphore et le procédé de reconstruction. 
Figure I

(métaphore de la lentille)

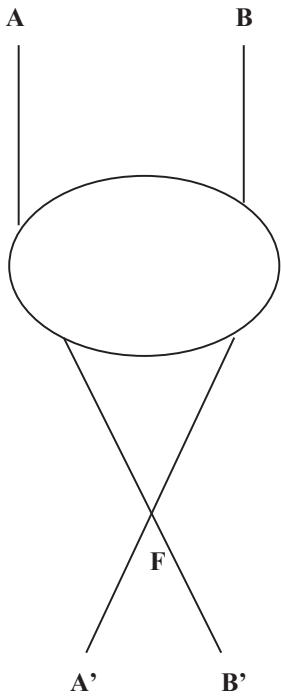

A et $\mathrm{B}=$ points des objets extérieurs

$\mathrm{F}=$ point de focalisation (où la relation spatiale disparaît)

$\mathrm{A}^{\prime}$ et $\mathrm{B}^{\prime}=$ points images

$\mathrm{A}$ " et $\mathrm{B} "=$ représentation spatiale de $\mathrm{A}$ et $\mathrm{B}$

\subsection{Formulation de l'hypothèse des signes locaux}

La théorie des signes locaux repose sur deux hypothèses distinctes, soit l'hypothèse physiologique qui concerne les mouvements de l'œil, et l'hypothèse psychologique qui porte sur le rôle des signes locaux dans la représentation et la localisation des objets dans l'espace. Suivant l'hypothèse physiologique, le signe local serait le résultat de la combinaison entre la sensation de couleur et les sensations musculaires qui accompagnent les mouvements de l'œil. Pour comprendre cette étrange combinaison entre sensations visuelles et sensations musculaires, quelques remarques élémentaires sur ce processus nerveux suffiront.

Il existe dans la rétine une partie appelée « tache jaune » (ou fovéa) qui possède une sensibilité à la lumière bien supérieure aux autres parties situées en périphérie, qui sont moins sensibles à la lumière. Lorsque la rétine est affectée par une vive lumière ou un point brillant sur sa partie latérale, le stimulus déclenche non seulement une sensation de couleur mais en même temps et de manière purement mécanique, une tendance à 
déplacer l'œil de manière à diriger le stimulus vers la vision fovéale ou le point de la plus claire vision. Car l'œil recherche l'excitant qui touche une place latérale de la rétine et pour le voir plus clairement, il déplace la tache jaune à l'endroit de la plus claire vision. Ce mouvement de l'œil est comparable à un mouvement réflexe en ce qu'il est involontaire et n'implique aucune conscience des actions musculaires de l'œil.

Ce mouvement oculaire diffère pour chaque point de la rétine, ou bien suivant la direction du mouvement, ou bien suivant sa grandeur. Appelons $X$ le point central où la vision est la plus claire, $p, q, r \ldots$ les points de la rétine qui sont excités et représentons-nous la rétine comme une surface sphérique. Ainsi, pour amener le point $X$ à la place du point $\mathrm{p}$, nous devons supposer une rotation de l'œil, $X_{p}$, qui est différente de toute autre rotation, provoquée par l'excitation q ou $\mathrm{r}$, qui occasionnent les mouvements de rotation $X_{q}$ et $X_{r}$ par exemple. Tous ces mouvements sont différents les uns des autres tant par leur grandeur que par leur direction. Par exemple, une excitation qui tombe sur une position latérale $p$, détermine une rotation de l'œil de la place $X$ à $p$. Pour chaque point $\mathrm{p}$, l'arc $X_{p}$ diffère de l'arc décrit par $X_{q}, X_{r}$, etc., et tous ces mouvements, décrits par les $\operatorname{arcs} X_{p}, X_{q}$ et $X_{r}$, ne diffèrent les uns des autres que par la grandeur et la direction. Par exemple, si les points $p, q, r$ se situent à égale distance de $X$, ils seront différents quant à leur direction. Pour Lotze, dire que $p$ et $q$ sont éloignés l'un de l'autre « signifie seulement qu'une certaine grandeur de mouvement est nécessaire pour diriger le regard sur l'un ou sur l'autre ; je ne perçois les diverses situations de ces points que précisément comme autant d'excitations à opérer ce mouvement » (Lotze, 1879 : 586). La figure suivant, que je tire également de l'ouvrage de Pastore (1971), illustre clairement les arcs décrits par les mouvements de l'œil.

Tous ces mouvements musculaires diffèrent les uns des autres par un caractère qui leur est propre. Mais puisque l'œil ne peut accomplir qu'un seul mouvement à la fois, pour rendre compte du fait que nous pouvons percevoir simultanément différentes locations ou points dans le champ visuel, le mouvement réel de l'œil ne peut suffire. C'est pourquoi Lotze a recours à l'hypothèse d'une « tendance au mouvement » qui est déclenchée par l'impression d'une place quelconque sur la rétine et qui est associée à un souvenir d'une opération «qui doit être exécutée pour que les excitations plus faiblement senties sur $\mathrm{p}$ et q donnent lieu à la plus claire et la plus forte perception » (Lotze, 1879 : 584). 
Figure II

Arcs décrits par les mouvements oculaires

Eil stationnaire

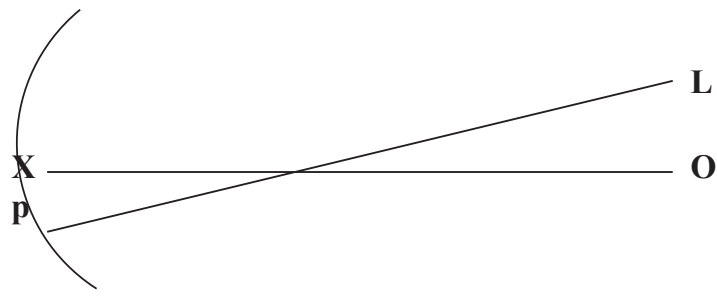

Eil en mouvement

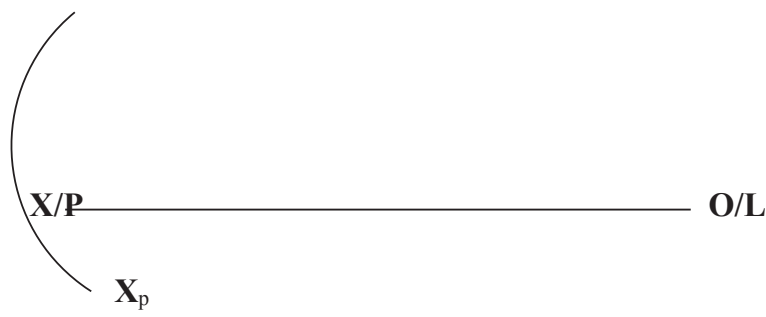

$\mathrm{X}=$ Fovea ou point de la plus claire vision

$\mathrm{L}=$ Point lumineux

$\mathrm{P}=$ Point de la rétine stimulé par $\mathrm{L}$

$\mathrm{X}-\mathrm{O}=$ État stationnaire de l'œil (avant l'effet exercé par L)

$\mathrm{X}_{\mathrm{p}}=\mathrm{L}$ 'arc décrit par le mouvement de l'œil pour amener la tache jaune $\mathrm{X}$ au point $\mathrm{p}$ où elle est stimulée

Ceci dit, ces mouvements oculaires et directions du regard ne sont pas des actes volontaires, ce sont des phénomènes physiques qui n'impliquent en définitive que le système nerveux. Or pour rendre possible la localisation, ces mouvements doivent agir de quelque manière sur nos états psychiques qui sont indispensables à la localisation. D'où l'hypothèse psychologique suivant laquelle ce sont les impressions que font naître ces mouvements ou les sentiments accessoires qui, à proprement parler, sont les véritables signes locaux. Stumpf l'a qualifié de théorie des stimulants psychiques parce que ces mouvements musculaires « causent, comme l'écrit Lotze, dans chaque fibre nerveuse, une impression accessoire particulière qui, pour chaque fibre, diffère de celle qu'éprouve toute autre, et s'unit, sous la forme d'une association, 
avec l'impression principale dépendante de la qualité de l'excitation, de sorte - par conséquent, - qu'aucune des deux n'altère la nature et le coloris spécifique de l'autre » (Lotze, 1879 : 571).

Ainsi, chaque stimulation produit, en plus d'une sensation de couleur, une sensation accessoire qui est indépendante de la couleur et de la nature de la place excitée sur la rétine. Cette impression locale $\pi, \chi, \rho$ est associée à une impression de couleur, par exemple à la couleur jaune $J$, de telle sorte que $J_{\pi}$ signifie un jaune qui agit sur le point $\pi, \mathrm{J}_{\chi}$ signifie le même jaune qui, selon le cas, agit sur le point $\chi$, et ainsi de suite. Ces impressions associées accessoirement fournissent en retour l'indice à partir duquel elle transpose le même jaune tantôt à un endroit, tantôt à un autre. Mais ces impressions accessoires doivent être complètement différentes de l'impression dominante (la couleur jaune) et elles doivent aussi différer les unes des autres de manière à ce que nous puissions assigner une place déterminée, à l'aide du signe local, à chaque impression individuelle d'un ensemble d'impressions données $p, q, r \ldots$

Par ailleurs, toutes les excitations $A, B, C$, qui touchent les parties de la peau ou de la rétine $p, q, r$, sont combinées avec des impressions accessoires $\pi, \chi, \rho$, qui sont les signes locaux, $\pi$ désignant la série entière des perceptions $\pi_{0} \pi_{1} \pi_{2} \ldots$ qui se succèdent durant la rotation de l'arc $X_{p}$. Ces impressions accessoires diffèrent les unes des autres et elles appartiennent à un système de signes, de telle sorte que pour chaque impression donnée, on puisse leur assigner, à l'aide d'un signe local, un lieu défini parmi tous les autres pour ainsi les localiser. Comme l'explique Lotze : " C'est de la combinaison des excitations de la rétine avec ces impressions inconscientes que leur associent dans l'âme les tendances au mouvement, qu'il faut déduire la coordination des points dans notre champ visuel » (Lotze, 1852, § 328). Cette coordination dans le champ visuel est assurée par un système de signes locaux qui émerge, en quelque sorte, d'un système de mouvements musculaires de l'œil, plus précisément de sensations de mouvement qui, elles, sont directement responsables de notre représentation des lieux (cf. Lotze, 1852: § 291 et Ribot, 1879 : 85-87).

\section{Les signes locaux et la controverse nativisme-empirisme}

L'originalité de la théorie de Lotze par rapport à celles de ses prédécesseurs réside entre autres choses dans deux hypothèses qui ont servi de base aux recherches sur la motricité durant la deuxième moitié du $\mathrm{XIX}^{\mathrm{e}}$ siècle. La première est la nature réflexe des mouvements de l'œil qui fournissent les signes locaux, c'est-à-dire l'appareil réflexe de l'œil qui répond aux stimuli de lumière dans la périphérie du champ visuel. Cette conception réflexe des mouvements s'est imposée à Lotze parce qu'il 
croyait que la relation entre la sensation de couleur et les mouvements de l'œil était établie dans le système nerveux et que les mouvements réflexes pouvaient lui fournir un système stable de signes locaux. Le deuxième trait distinctif de cette théorie est qu'elle remplace les mouvements actuels de l'œil par des tendances au mouvement (cf. Scheerer, 1984).

En adoptant une version de la théorie des signes locaux de Lotze, Helmholtz et Wundt ont aussi repris ces deux hypothèses sur la motricité. Helmholtz estime en effet que les sensations visuelles et tactiles diffèrent des autres sensations non seulement par leur intensité et leur qualité (couleur), mais encore par leur signe (local). Wundt, pour sa part, soutient dans sa Psychologie physiologique que l'intuition de l'espace et nos représentations les plus originaires de l'espace résultent de notre représentation du mouvement. Mais puisque la représentation du mouvement n'explique pas à elle seule notre capacité de localiser les objets dans l'espace lorsque l'organe tactile est au repos, elle doit être combinée avec la théorie des signes locaux de Lozte (cf. Lotze, 1879 : 592 sur les signes locaux tactiles). L'hypothèse de Wundt se ramène donc à l'idée que notre représentation de l'espace résulte en fait de la synthèse par association des sensations du mouvement et des signes locaux. D'où le privilège qui revient dans sa psychologie aux représentations tactiles et aux représentations de mouvement sur toutes les autres représentations sensorielles et l'importance qu'il accorde à la localisation tactile dans sa propre théorie des signes locaux.

Ceci dit, la controverse nativisme-empirisme par laquelle Helmholtz caractérise son différend avec Hering porte sur le thème plus général de la perception sensible. En première approximation, ce débat porte sur la question de savoir si la perception de l'espace est acquise par apprentissage et au moyen d'opérations mentales, comme le soutiennent les empiristes, ou si cette capacité est inhérente à la structure même de l'expérience sensible, comme le veulent les nativistes. Pour bien comprendre les enjeux de ce débat, une brève présentation des grandes lignes de la théorie empiriste de la perception s'impose (voir figure III). 


\section{Figure III}

Théorie classique de la perception

\section{OBJETS}

(Stimuli distants)

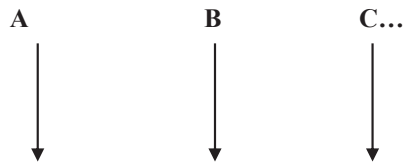

Stimuli proximaux

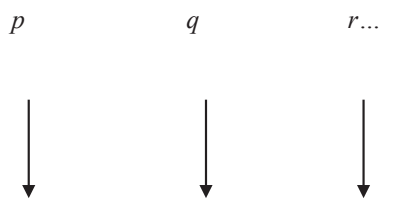

Impressions/ réponses sensorielles

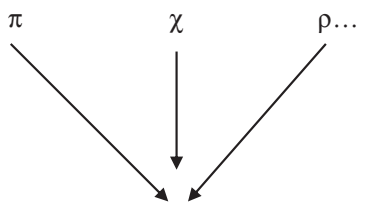

PERCEPTION

(Associations, inférences ou chimie mentale)

Les stimuli distants désignent ici les objets de notre environnement, et plus précisément les propriétés physiques de ces objets (la distance, la position dans l'espace, le volume, la forme, etc.) qui affectent notre vision au moyen de la lumière qu'elles réfléchissent sur les récepteurs de la rétine. L'énergie de lumière ou de son qui agit directement sur nos organes sensibles produit des stimuli proximaux. Cette notion désigne l'image ou pattern de la rétine qui contient des indices qui renvoient aux propriétés des objets physiques et de leur environnement. La lumière, qui est réfléchie par le stimulus distant devant l'œil, passe par le foyer de la lentille de l'œil et agit sur les cellules sensibles à la lumière sur la rétine située derrière l'œil. L'image ainsi projetée sur la rétine et qu'on appelle aussi un pattern de stimulation est analysée par les récepteurs sensoriels et ce qu'on appelle aussi le processus d'encodage, qui produit la réponse sensorielle. 
Après Lotze, Helmholtz soutient que les sensations visuelles et tactiles ne diffèrent pas seulement des autres sensations par leur intensité et leur qualité (couleur), mais qu'elles présentent une troisième différence, qui dépend de la partie de la rétine ou de la peau qui est affectée et qu'il appelle un signe (local). D'où le premier principe de l'empirisme de Helmholtz, qu'il formule de la manière suivante : "Les sensations sont, pour notre conscience, des signes dont l'interprétation est livrée à notre intelligence » (Helmholtz, 1867 : 797). Pour Helmholtz, les sensations sont comparables à des signes qui renvoient à des propriétés spatiales ou temporelles du monde extérieur, mais ces signes doivent être interprétés et cette interprétation doit être acquise au moyen de l'expérience. Ces signes n'acquièrent donc un rapport à l'espace que par une interprétation psychologique. Aux conditions physiologiques de la localisation s'ajoutent donc les conditions psychologiques qui, dans la figure III, rendent possible le passage des données sensorielles à la perception proprement dite et permettent ainsi de combler le fossé entre les sensations brutes et la perception des objets du monde. Ainsi, la tâche de cette partie de la physiologie optique que Helmholtz appelle psychologie est d'étudier les processus par lesquels les sensations non spatiales sont combinées pour former des représentations de l'espace. C'est ce qu'on appelle la théorie de la projection : les images perceptuelles des objets sont projetées dans l'espace au moyen de processus mentaux. Ces processus sont à concevoir, comme l'explique Helmholtz dans son Handbuch (Helmholtz, 1867 : 447, 453), sur le modèle des inférences dans la logique de J. S. Mill dont l'idée d'une « chimie mentale » a aussi influencé Wundt. Supposons une sensation donnée de lumière dans certaines fibres du mécanisme nerveux de la vision. Voir un objet lumineux dans notre champ de vision serait le résultat d'une inférence qui nous permet de conclure, sur la base d'une telle sensation donnée, à l'existence d'un objet dans notre champ de vision. Ces inférences sont inconscientes et de nature inductive, tandis que ces inductions sont fondées sur la loi de la causalité.

Le différend qui oppose Helmholtz à Hering porte justement sur le recours à ces éléments psychologiques dans l'explication de la perception sensible. Ce problème ressort clairement dans la théorie des couleurs qu'il est maintenant convenu d'appeler la théorie Young-Helmholtz. Helmholtz a montré qu'en choisissant un ensemble de trois longueurs d'onde correspondant à celles des couleurs rouge, vert et bleu, nous pouvions, en les combinant selon différentes proportions, produire n'importe quelle autre couleur. Ce procédé permettait de simplifier considérablement le travail de la physiologie en la réduisant à l'étude de la manière dont nous percevons ces trois couleurs. Il était ensuite possible d'expliquer tout le reste à l'aide des combinaisons possibles entre ces trois éléments de base, et c'est justement ce qu'a cherché à faire 
Helmholtz. Selon lui, il y aurait trois types de fibres nerveuses dans l'œil. L'excitation d'un type produirait la sensation de rouge, la seconde de vert et la troisième de bleu. La lumière exciterait tous ces trois types de fibres avec une intensité qui varie selon la longueur d'onde de la lumière. Les fibres, qui sont sensibles au rouge, seraient excitées pour la plupart par la lumière de l'onde la plus longue ; celles qui sont sensibles au violet seraient excitées par la lumière de l'onde la plus courte, et ainsi de suite. La question était alors de savoir si cette explication des couleurs pouvait être transposée aux autres qualités du monde de la perception, et d'abord à celles de la taille d'un objet, de sa position, sa distance, sa forme, etc. La réponse est simple : il n'existe aucune « énergie spécifique » au moyen de laquelle nous pourrions rendre compte des caractéristiques spatiales, il n'existe aucune structure anatomique qui soit sensible à la profondeur, à la distance, aux objets et à la forme. Dans ces conditions, la physiologie de l'époque faisait face à l'alternative suivante : ou bien nier qu'il y ait de telles structures anatomiques et adopter le point de vue empiriste qui offrait une option pour combler le fossé entre les organes sensoriels périphériques et le monde des objets ; ou bien rechercher des mécanismes sensoriels et faire le pari qu'il existe en effet de telles structures pouvant rendre compte de la distance, par exemple. Helmholtz a donc opté pour la première, Hering pour la deuxième option (cf. Turner, 1994).

Ces mécanismes sensoriels, auxquels Hering a recours devant le problème de la localisation des objets dans l'espace et celui de la vision binoculaire, représentent le cœur de ce qu'on appelle sa théorie des valeurs spatiales et rétiniennes. Cette théorie, qui est en fait une autre extension de la théorie de signes locaux de Lotze, veut que la rétine possède des mécanismes physiologiques inhérents pour évaluer la disparité des images et les convertir en perceptions spatiales. Ainsi, chaque point sur la rétine fournit, en plus des sensations de lumière et de couleur, trois sensations d'espace ou trois qualités spatiales sensibles séparées qui sont appelées valeurs spatiales (Raumwerte) : une « valeur » de hauteur, de largeur et de profondeur. Ainsi, lorsqu'un point de la rétine est stimulé, nous percevons immédiatement l'image comme étant située en dessous ou au-dessus, à droite ou à gauche, d'un point de fixation, avec la distance de ce point déterminée par la valeur de hauteur et de largeur du point particulier qui est stimulé. Si l'on accepte la théorie de Hering, il faut alors admettre que notre expérience de la distance est aussi immédiate que celle de la couleur rouge, par exemple. Je n'entrerai pas ici dans le détail de la théorie de Hering et ne commenterai pas non plus les réponses de Helmholtz afin de mieux me concentrer sur l'aspect philosophique du débat.

Dans ses remarques préliminaires à Zur Lehre vom Lichtsinne, Hering s'adresse directement à Helmholtz et s'oppose à la direction prise par les recherches dans le domaine de la physiologie des sens et dans la 
physiologie de l'optique de Helmholtz en particulier. Il conteste les termes mêmes de l'opposition par laquelle Helmholtz cherche à caractériser le débat. À propos de la caractérisation de sa théorie de la vision binoculaire en termes de nativisme, Hering remarque au début de son ouvrage qu'elle n'est pas appropriée puisqu'il s'agit alors d'un aspect accessoire de leur opposition. Parce qu'entre le nativisme et l'empirisme, il n'y a qu'une différence de degré tant et aussi longtemps qu'on demeure sur le sol de la physiologie, notamment tant que l'on s'en tient à la méthode physiologique. L'objet principal du litige porte au contraire sur le recours à la psychologie empiriste. C'est ainsi que Hering lui reproche de se commettre à une forme de spiritualisme - entendons par là une forme de mentalisme - en ce qu'il répond par la psychologie à des questions qui, selon Hering, peuvent être traitées avec succès par la physiologie. Comme l'écrit Hering : « ce qu'on ne voulait ou ne pouvait pas rechercher physiologiquement, on l'expliquait depuis une "Lebenskraft", ce qui explique alors qu'à toutes les trois pages d'un traité de physiologie optique figurent, à la manière d'un deus ex machina, les mots "âme" ou "esprit", "jugement" ou "inférence", afin de se débarrasser de toutes les difficultés » (Hering, $1878: 2$ ).

En résumé, la critique de Hering porte sur deux aspects de la théorie de la perception sensible de Helmholtz. Le premier aspect concerne le recours à des inférences inconscientes dans l'explication de phénomènes comme la perception de l'espace que Hering croit pouvoir expliquer en ayant recours uniquement à la physiologie. C'est pourquoi le cœur du litige réside, selon le diagnostic de Hering, dans une différence de méthode : utiliser la nature de l'esprit humain comme principe d'explication dans le domaine de la physiologie, c'est se rendre coupable d'une erreur de catégorie (Hering, $1878: 4$ ). D'autre part, ne perdons pas de vue que cette critique du mentalisme de Helmholtz n'entraîne aucunement le rejet de toute forme de psychologie dans le domaine de la physiologie. Hering en a d'abord et avant tout contre la psychologie empiriste de l'association et ses postulats sensualistes (sensations brutes), mentalistes (le recours à des processus mentaux), intellectualistes (les processus qui lient perceptions et sensations sont de nature judicative et sont associatifs) et mécanistes (ces processus sont des inférences inconscientes puisqu'ils opèrent sur des sensations non remarquées et sans que le sujet percevant en soit conscient de quelque manière). La psychologie telle que l'entend Hering se compare davantage à ce que Stumpf appelle la phénoménologie dont nous reparlerons plus tard. 


\section{Stumpf et la théorie des signes locaux}

Les deux objections principales que Stumpf adresse à la théorie des signes locaux de Lotze concernent la notion de stimulant psychique et les présupposés métaphysiques dans sa conception générale de l'espace.

\subsection{Critique de la notion de stimulant psychique}

Stumpf soutient en effet que cette sensation auxiliaire de mouvement (Mitempfindung), que Lotze conçoit comme un motif incitant l'âme à appliquer sa tendance spatialisante à des contenus individuels de sensation, est, en tant que telle, le signe local du sens visuel dont la fonction est de motiver ou de produire dans l'âme la représentation du lieu déterminé auquel renvoie ce signe (ou son lieu d'origine). La question que pose Stumpf dans son Raumbuch porte justement sur le mécanisme par lequel les signes locaux rendent possible la transformation des données intensives en données extensives, c'est-à-dire en représentations locales.

Stumpf (1893) distingue deux versions de la théorie des signes locaux de Lotze. Suivant la première, les mouvements ou tensions des muscles oculaires agiraient comme des stimulants physiques et causeraient une impression inconsciente dans l'âme, laquelle serait directement responsable de la représentation du lieu. D'après cette interprétation, les signes locaux ne seraient en définitive que des phénomènes physiques et le processus de localisation serait comparable aux mouvements réflexes qui opèrent « à notre insu » ou de manière inconsciente (cf. Lotze, 1852 : $\S 294)$. Cette interprétation correspond à la version présentée par Lotze dans sa Psychologie médicale et elle est rejetée explicitement dans son article de 1877, « De la formation de la notion d'espace », dans lequel il propose une deuxième version de sa théorie. Dans cet article, Lotze écrit : "Ce qui se passe dans les nerfs ne peut servir de mobile qu'à une rotation, c'est-à-dire à un phénomène du monde physique ; les affections psychiques, qui en proviennent, méritent seules le nom de signes locaux, car elles seules peuvent provoquer la localisation » (Lotze, $1877: 72$ ). La question est alors de savoir quelle est la nature de ces affections psychiques et quel est le sens de ce qu'il appelle ici le motif par lequel le signe motive ou déclenche la représentation locale. Dans son commentaire sur cet article de Lotze, Stumpf (1893: 71) a vu dans ce passage une confirmation de l'interprétation qu'il propose dans son Raumbuch et suivant laquelle le signe local de Lotze n'est ni le lieu de la sensation dans l'espace visuel, ni un index local, mais bien la cause psychique de cette localité, c'est-àdire ce qu'il appelle aussi un stimulant (Reiz) psychique. Par stimulant psychique, il entend le processus qui intervient entre les mouvements de l'œil et les représentations de lieu, et Stumpf conçoit cet intermédiaire en termes de stimulation psychique et de causalité psychique (cf. Stumpf, 
1873 : 93). Contrairement à la sensation de couleur déclenchée directement par l'excitant physique, il y aurait donc, entre la sensation de lieu et l'excitant physique, un intermédiaire psychique qui agirait, comme dans la causalité physique, en tant que stimulant psychique (et sensation de mouvement), et qui aurait pour conséquence immédiate la représentation d'un lieu déterminé et toujours le même.

Stumpf se demande si l'usage que fait Lotze de la notion de signe local pour désigner à la fois la sensation de mouvement et la cause de la représentation de lieu est approprié. Admettons que le signe local fonctionne comme un signe indicatif (Merkzeichen) et qu'il agit à la fois comme renvoi d'un signifiant à un signifié, comme la fumée au feu, et comme motif psychologique dans l'association de la présence de la fumée à l'existence du feu. La connaissance du désigné au moyen d'un signe local reposerait alors sur l'association et la reproduction. Mais, remarque Stumpf (1873: 95), cette relation diffère d'une relation causale à la fois parce qu'elle est arbitraire (le signe comme les relations associatives entre la fumée et le feu sont arbitraires), ce qui ne convient pas à la relation entre représentation de l'espace et sensation de mouvement qui, suivant la théorie du stimulant psychique, est censée être fixe et toujours la même. D'autre part, si l'on voulait utiliser le modèle de la causalité physique dans le domaine du psychique pour rendre compte de la relation entre les sensations musculaires et la représentation locale, ça ne fonctionnerait pas non plus parce que la relation causale entre le stimulant psychique et la représentation spatiale est univoque en ce sens que le signe ne peut être à la fois cause et effet, effet de stimulations externes et cause de stimulations internes. Or pour que le signe local puisse fonctionner comme une cause, il faudrait qu'il soit non arbitraire et permutable, ce qui ne convient pas à sa vocation initiale.

\subsection{Critique de la présupposition métaphysique dans la théorie de Lotze}

L'hypothèse de Lotze de sensations auxiliaires ou quasi-sensations ne concerne en définitive que la localisation des sensations dans le champ visuel et tactile suivant leur lieu d'origine, mais elle n'explique pas « cet ordre premier des sensations » que présuppose l'hypothèse de signes locaux. Autrement dit, la question à laquelle Lotze cherche à répondre avec sa théorie des signes locaux n'est pas la question métaphysique du statut de l'espace en général, mais bien celle de la localisation des sensations qui, parce qu'elles ne sont pas en soi étendues, présupposent un espace objectif. C'est d'ailleurs ce qu'il reconnaît en distinguant le problème de la localisation ( « comment fait l'âme pour assigner dans cette intuition de l'espace, qui lui est nécessaire, à chacune de ses sensations sa place déterminée, en correspondance avec l'objet qui en est la cause ») 
du problème métaphysique des représentations spatiales qui répond à la question de savoir « pourquoi l'âme arrange la multitude de ses sensations dans ce cadre de relations géométriques » et non dans un autre ordre, pourquoi cette forme d'intuition et pas une autre (Lotze, 1877 : 352).

Comme l'a bien vu Wundt (1874: 36 et 1878), si l'hypothèse de Lotze doit pouvoir être utilisée efficacement dans le domaine des représentations de l'espace, nous devons d'abord écarter ses présupposés métaphysiques, c'est-à-dire les présupposés d'un espace kantien compris comme une forme a priori du sens externe. Wundt comme la plupart des philosophes et scientifiques après Herbart étaient d'avis que le domaine d'étude qualifié par Kant d'esthétique transcendantale n'est pas un postulat métaphysique vide, mais bien un champ d'investigation commun aux sciences empiriques et à la philosophie. Tel est aussi un des facteurs déterminants dans le développement de la nouvelle psychologie tout comme dans l'émergence de la phénoménologie comprise comme le domaine des phénomènes sensibles.

Stumpf est d'accord avec Wundt sur ce point, mais il ajoute qu'il faut aussi s'attaquer à une autre présupposition que véhiculent le kantisme et l'empirisme de Wundt, à savoir la dichotomie traditionnelle de la forme et de la matière. La matière est définie dans la première Critique comme ce qui correspond à la sensation dans le phénomène, tandis que la forme du phénomène correspond à ce qui fait que la multiplicité des phénomènes est ordonnée suivant certaines relations. Stumpf fait valoir que cette distinction épistémologique a nui considérablement au développement de la psychologie et qu'elle est directement responsable de nombreux Scheinprobleme dans tous les domaines où elle a été appliquée (cf. Stumpf, 1891). D'un côté, Kant conçoit les sensations comme un matériau informe et non structuré servant de support à l'activité de l'entendement, ce qui revient à dire, comme l'écrit Stumpf, que pour les kantiens « nos sensations ne présentent en soi aucune caractéristique des différences spatiales, par exemple que des couleurs apparaissent ponctuellement et que leur extension en lignes et surfaces serait déjà l'œuvre d'une activité de l'esprit » (Stumpf, 1907 : 186-187). D'un autre côté, l'espace kantien est un principe d'ordonnancement ou un " cadre de relations géométriques » imposé à cette multiplicité sensible. Stumpf fait valoir au contraire que l'espace n'est pas à l'origine un principe d'ordonnancement ou de classification parce qu'un tel principe, dans le cas de l'espace, présuppose une représentation plus originaire de l'espace dont le contenu fonde cet ordre spatial.

Un des principes importants que Stumpf oppose au kantisme dans son Raumbuch est qu'il n'y a pas d'espace sans qualités sensibles et on ne peut donc pas se représenter l'espace sans une qualité sensible 
quelconque, comme on ne peut se représenter une qualité quelconque sans une forme. Stumpf vise ainsi le deuxième argument de Kant dans son esthétique transcendantale en faveur de l'origine subjective de l'espace et de son statut de forme subjective, argument suivant lequel nous pouvons nous représenter l'espace sans les qualités, bien qu'il soit impossible de se représenter les qualités sans l'espace. Stumpf conteste le bien-fondé de cette distinction entre qualité et matière en faisant valoir que l'on ne peut absolument pas se représenter l'espace sans la qualité, comme par exemple un carré sans un fond de couleur et « qu'il est impossible de représenter l'espace, l'étendue, la forme sans une qualité sensible quelconque » (Stumpf, 1891 : 483). On peut certes faire abstraction de la qualité comme par exemple dans la formation d'un corps géométrique ; mais il ne s'agit pas alors de la représentation d'un corps qui possède uniquement l'étendue et aucune autre propriété, mais d'un corps dont nous ne prenons en considération que les relations spatiales en faisant abstraction de ses autres propriétés qui n'obéissent pas aux lois de la géométrie. Mais l'espace et les rapports spatiaux ne sont pas séparés et indépendants des qualités - on ne peut se représenter un espace vide (Stumpf, $1873: \S 1$ ). De ce point de vue, l'espace kantien est une abstraction en un double sens : d'abord au sens où il idéalise la conception classique d'un espace sans qualité, homogène, isotrope et infini, et ce par opposition à l'espace sensoriel ou phénoménal qui est indissociable des qualités sensibles et qui est fini. Il est aussi abstrait dans un deuxième sens, à savoir qu'il s'agit d'un concept et plus précisément d'une formation conceptuelle qui résulte d'un processus d'abstraction fondé sur la perception sensible. Stumpf soutient en effet que tous les concepts, y compris le concept d'espace, sont des abstracta qui tirent leur origine de la perception sensible, ce sont des " entia rationis cum fundamento in re » (Stumpf, 1939 : 24). Dans cette perspective, l'espace kantien est tout simplement un abstractum et pas du tout une intuition, l'espace n'étant pas une forme a priori mais plutôt une formation conceptuelle a posteriori (cf. Stumpf, $1906: 73$ ).

\section{La position de Stumpf dans ce débat}

Comme l'explique Stumpf dans son Raumbuch, si l'hypothèse de Lotze a pu faire consensus autant chez les empiristes que chez les nativistes, c'est qu'elle se situe à mi-chemin entre deux positions irréconciliables. C'est ce que montre la classification que propose Stumpf dans cet ouvrage des positions et points de vue sur la localisation et la nature de la représentation de l'espace (cf. Woodward, 1978). Elle se situe à mi-chemin entre l'empirisme et le nativisme en ce que Lotze admet, avec les empiristes, que ce que nous percevons originairement, ce sont des qualités avec intensité différente et qu'on ne peut en dériver les représentations spatiales ; mais il admet aussi, avec les nativistes, que ces 
dernières ne sont pas le résultat d'un processus associatif. Stumpf estime que si l'on doit pouvoir conserver la théorie des signes locaux de Lotze, c'est au niveau strictement physiologique et à condition d'abandonner l'idée de stimulations psychiques. La position que défend Stumpf dans son Raumbuch est en fait la contrepartie psychologique du nativisme de Hering dans le domaine de la physiologie, position que l'on peut qualifier dans les circonstances de nativisme modéré parce qu'elle admet que des processus psychiques interviennent dans la détermination des différences de profondeur, par exemple, et elle s'oppose à un nativisme extrême qui, lui, exclut a priori tout recours à quelque processus psychique que ce soit. Mais elle n'a rien à voir avec ce " faux nativisme des idées innées » qui statue sur des facultés innées de l'âme.

Dans son Raumbuch, Stumpf pose dès le départ que ce que nous percevons originairement et directement, ce sont ni les sensations, comme le pensent les empiristes, ni l'espace un et infini des kantiens, mais bien le Sehraum : "Il y a un espace visuel, à savoir un contenu sensible particulier qui est ressenti directement de la même manière que la qualité de couleur en conséquence du processus nerveux optique et il porte en lui tous les traits caractéristiques que nous attribuons à l'espace » (Stumpf, 1873 : 272). L'espace est compris dans le Raumbuch comme un contenu de représentation qui peut être perçu au moyen de plusieurs sens (notamment le sens de la vue et du toucher) et il constitue, avec une qualité sensible (notamment la couleur et le toucher), un contenu inséparable (Stumpf, $1873: 7)$. Nous pouvons certes faire abstraction de la couleur et nous pouvons aussi nous représenter une couleur de plus en plus petite, mais tout contenu visuel est déterminé spatialement comme il est aussi déterminé temporellement, qualitativement et intensivement. Dans cette perspective, la manière même de poser la question de l'origine de la perception de l'espace n'est pas, comme le pensaient les kantiens et les empiristes, de savoir comment nous lions espace et qualité sensible, mais bien de savoir de quelle manière nous parvenons à les distinguer. Et contre Lotze, ce ne sont donc pas nos représentations des lieux individuels qui, au moyen de signes indicatifs, seraient coordonnés à l'ensemble du champ visuel, mais bien l'inverse : c'est le champ visuel qui est d'abord représenté et les qualités sont ensuite ordonnées en lui (cf. Stumpf, 1873 : 79 sq., 89, 146). La perception du tout ou du contenu unitaire prime donc sur la perception de ses parties comme un orchestre sur ses instruments, une mélodie sur ses notes, une odeur sur ses parfums ou encore le goût d'un mets quelconque sur ses saveurs.

La position que défend Stumpf dans son Raumbuch s'appuie sur l'idée que la couleur et l'étendu forment un tout indissociable qui se fonde sur les relations de dépendance des contenus partiels. Tel est le principe qui guide Stumpf dans sa critique de l'empirisme et du kantisme, comme dans 
sa défense du nativisme. L'espace et la qualité de couleur forment un seul et même contenu dont ils sont les contenus partiels (Theilinhalte). Par « contenus partiels », Stumpf entend « des contenus indépendants [qui] sont donnés là où les éléments d'un complexe de représentation peuvent aussi être représentés, suivant leur nature, de manière séparée ; contenus partiels là où ce n'est pas le cas ». Stumpf distingue deux grandes classes de relation entre les contenus de représentation : la classe des contenus indépendants, comme la couleur et le son qui sont des qualités spécifiques à des sens différents ; et la classe des relations de dépendance dont les termes, comme la qualité et l'étendue, ne peuvent être représentés de manière séparée. Les relata d'une relation de dépendance « ne peuvent, suivant leur nature, exister séparément les uns des autres, ils ne peuvent être représentés séparément 》(Stumpf, 1873 : 114). Autrement dit, on ne peut percevoir un contenu purement qualitatif (couleur, son) ou quantitatif (intensité), c'est-à-dire une substance sans attributs. Le critère le plus simple pour distinguer ces deux classes de relation réside dans la possibilité, dans le cas d'une relation d'indépendance, de nous représenter séparément deux contenus comme l'étendue et la couleur, par exemple, soit en imagination, soit au moyen de l'expérimentation. Lorsqu'on réussit à se représenter deux contenus séparément, comme lorsque nous avons affaire à des sensations appartenant à des champs sensoriels différents, ce sont alors des contenus indépendants ; dans le cas contraire, ce sont des contenus dépendants. Mais ces relations de dépendance ne sont ni associatives, ni sommatives, ce sont des relations nécessaires qui obéissent à ce qu'il appelle des lois de structure. Et ces relations ne sont pas des concepts, catégories ou Denkformen a priori imposés aux sensations de l'extérieur par un acte ou une opération mentale quelconque, elles appartiennent intrinsèquement aux phénomènes sensibles.

L'espace senti originairement a trois dimensions. Chaque contenu spatial que nous représentons est nécessairement représenté dans une certaine distance ou profondeur. Ce qu'on appelle direction d'un point vue en rapport à la profondeur est son lieu au sein d'une surface vue en profondeur (Stumpf, $1873: \S 12$ ). Notre représentation de l'espace présuppose en outre un rapport à un certain centre naturel, qui lui est extérieur, et ce centre naturel n'est rien d'autre que la position du corps propre. Ainsi, la distance, le lieu, la grandeur, la direction d'un objet ou encore dans les déterminations du " l'un à côté de l'autre », de la droite ou la gauche, etc. présupposent toujours une relation à la position locale du corps propre (Stumpf, 1873 : 180). Cette relation n'est pas rajoutée, mais elle est nécessairement et originairement inhérente à la détermination individuelle du lieu et elle ne peut donc pas être séparée de sa représentation. Cependant, la profondeur perçue et l'évaluation précise 
de la distance requièrent l'apport du concept et d'opérations psychiques que Stumpf appelle des fonctions.

\section{Remarques finales}

$\mathrm{Au}$ tout début de cette étude, j'ai suggéré que cette controverse sur la perception de l'espace a mis en place les conditions propices au développement de la jeune phénoménologie. Pour terminer, je voudrais étayer ce point à l'aide de quelques remarques de Stumpf et de son étudiant Husserl qui vont dans ce sens. Ma première remarque concerne l'origine de la méthode phénoménologique et elle est tirée des Conférences d'Amsterdam de Husserl dans lesquelles il affirme que sa phénoménologie peut être comprise comme « une certaine radicalisation d'une méthode phénoménologique développée et pratiquée auparavant déjà par certains chercheurs des sciences de la nature et certains psychologues » (Husserl, 1962 : 302). Husserl indique au même endroit deux Naturforscher qui auraient pratiqué la méthode phénoménologique, soit Ernst Mach et Ewald Hering, de même que Brentano (Fisette, 2009b ; 2010). La thèse de l'origine de la méthode phénoménologique chez Mach et surtout Hering a aussi été défendue par Stumpf qui a utilisé lui aussi le terme " phénoménologie » pour désigner le domaine d'étude des éléments ou phénomènes sensibles, et il attribue lui aussi la paternité de cette méthode phénoménologique à Hering. Dans la deuxième section de son article de 1917 sur les attributs du champ visuel, Stumpf attribue à Hering le mérite d'avoir introduit la méthode phénoménologique dans le domaine de la perception sensible : « Si quelque chose doit valoir de manière complète et définitive quant aux efforts de Hering, c'est l'exigence d'un point de départ psychologique, voire phénoménologique, dans la théorie des couleurs. La clarté de ses développements concernant l'ingérence néfaste du point de vue physicaliste dans la description des phénomènes sensibles demeure à jamais exemplaire » (Stumpf, $1917: 7$ ).

Le point de départ de Hering dans la description des Sehdinge ou phénomènes sensibles n'est pas arbitraire puisqu'il représente un domaine propre de recherche. La science ou la discipline qui sert de propédeutique à la science et dont la tâche consiste dans l'étude de ce domaine est justement cette phénoménologie qui se définit comme l'analyse des phénomènes sensibles dans ses éléments derniers. Ce domaine d'étude représente le point de départ commun à toutes les sciences, et il s'oppose au point de départ physicaliste dans les stimulations (Reize) extérieures, comme le préconise Helmholtz, par exemple. Le problème des attributs du champ visuel dont traite Stumpf dans son traité de 1917 est un problème qui relève avant tout de la phénoménologie pour autant qu'elle cherche à fournir une description complète d'un genre de phénomènes 
sensibles et étudie les lois structurelles inhérentes aux phénomènes sensibles en général. D'où le mérite qu'il attribue à Hering d'avoir reconnu l'importance d'une étude préalable des phénomènes et d'avoir ainsi conféré au domaine de la phénoménologie un statut privilégié par rapport à celui des autres sciences. En fait, en privilégiant ce point de départ, Hering aurait reconnu le primat de la phénoménologie sur la physiologie. À cet égard, écrit Stumpf, «l'offre est toujours du côté de la phénoménologie, et la demande du côté de la physiologie » (Stumpf, 1906 : 31). En d'autres mots, la description du «perçu » précède son explication physiologique ou neurologique et détermine même cette dernière, puisque c'est l'analyse descriptive ou phénoménologique qui est du côté de l'offre en ce qu'elle fournit à une science comme la physiologie, la demanderesse, son explanandum ${ }^{2}$.

On pourrait montrer que cette problématique sur l'origine de la perception de l'espace et les signes locaux est au cœur des préoccupations du jeune Husserl durant ses études à Halle auprès de Stumpf. En effet, dès sa première année d'étude à Halle, Husserl s'est vu imposer un examen visant à reconnaître son diplôme étranger d'Autriche. Dans le jury siégeaient le mathématicien Cantor et, ex officio, Stumpf qui l'a examiné entre autres choses sur le thème de la théorie des signes locaux de Lotze, l'histoire des théories de l'espace et les relations entre la logique et les mathématiques (cf. Fisette, 2009c). C'est aussi durant cette période que Husserl a annoté systématiquement le Raumbuch de son mentor Stumpf. Husserl a laissé derrière lui plusieurs manuscrits de recherche datés du début des années 1890 qui appartiennent pour la plupart au projet d'un Raumbuch qui devait faire partie du deuxième volume de Philosophie de l'arithmétique qui n'a jamais vu le jour. Ces manuscrits de recherche nous permettent de situer de manière assez précise la position adoptée par Husserl dans ce débat sur la perception de l'espace. On remarque en effet le penchant de Husserl pour une position nativiste, très proche d'ailleurs de celle de Stumpf, comme le montre notamment l'important fragment 10 où il examine ce débat et dans lequel il est question des signes locaux de Lotze (Husserl, 1983 : 269, 309, 306 sq.). Une partie de ces recherches a mené à la rédaction de son article de 1894, «Études psychologiques pour la logique élémentaire », dans lequel les travaux de

2 Hering a comparé sa méthode à celle utilisée dans le forage d'un tunnel et cette analogie a été reprise par Mach dans son ouvrage Connaissance et erreur afin d'illustrer la méthode descriptive qui caractérise sa propre recherche dans le domaine des éléments (Mach, 1906 : 14). Notons également que Stumpf et Husserl ne sont pas non plus les seuls à faire le rapprochement de Hering et Mach avec la méthode phénoménologique. Plusieurs étudiants de Husserl durant la période de Göttingen ont vu dans la phénoménologie de Husserl un cas particulier de la méthode pratiquée par Hering puis par Mach. Parmi les plus importants, mentionnons David Katz (1911), H. Hofmann (1913), Paul F. Linke (1918) et Éric Jaensch (1927). 
Stumpf et de Lotze sur la perception de l'espace sont d'ailleurs qualifiés de « recherches magistrales » (Husserl, 1894 : 162). C'est aussi dans ce contexte que Husserl a pris conscience de l'importance de la théorie des touts et des parties de Stumpf qu'il reprend à son compte dans cet article de 1894 et qu'il développe systématiquement dans la troisième de ses Recherches logiques.

\section{Bibliographie}

Ackerknecht, E., Theorie der Lokalzeichen, Tübingen, Mohr, 1904.

Fisette, D., "Carl Stumpf (1848-1936) ", in Stanford Encyclopedia of Philosophy, http://plato.stanford.edu/entries/stumpf/, (02.03.2009), 2008.

-, "Stumpf and Husserl on Phenomenology and Descriptive Psychology », Gestalt Theory 31, 2, 2009a, p. 175-190.

-, «Fenomenologia e fenomenismo in Husserl e Mach », Scientice Studia, 7, 4, 2009b, p. 535-76.

-, « Husserl à Halle (1886-1901)», Philosophiques, 36, 2, 2009c, p. 277-306.

-, « Descriptive Psychology and Natural Sciences. Husserl's Early Criticism of Brentano ", in C. Ierna, H. Jacobs, F. Mattens (eds.), Edmund Husserl 150 Years : Philosophy, Phenomenology, Sciences, Berlin, Springer, 2010, p. 135167.

Fisette, D. (ed.), Carl Stumpf. Renaissance de la philosophie, Paris, Vrin, 2006.

Fisette, D.; Martinelli, R. (eds), Philosophy from an Empirical Standpoint. Essays on Carl Stumpf, Amsterdam, Rodopi, 2015.

Helmholtz, H. von, Handbuch der physiologischen Optik, 3 Bde. Leipzig, L. Voss, 1867.

Hering, E., Die Lehre von binoculären Sehen, Leipzig, Engelmann, 1868.

Hering, E., Zur Lehre vom Lichtsinne. Sechs Mitheilungen, Wien, Akademie der Wissenschaften in Wien, 1878.

Hofmann, H., « Untersuchungen über den Empfindungsbegriff », Archiv für die gesamte Psychologie, 2, 1913, 1-136.

Husserl, E., Phänomenologische Psychologie. Vorlesungen Sommersemester 1925, Husserliana Bd. IX, Den Haag, Nijhoff, 1962.

-, Philosophie der Arithmetik, Husserliana Bd. XII, Den Haag, Nijhoff, 1970.

-, « Études psychologiques pour la logique élémentaire » [1894] in Articles sur la logique, Paris, PUF, 1975, 123-167.

-, Studien zur Arithmetik und Geometrie. Texte aus dem Nachlass (1886-1901), Strohmeyer, I. (ed.), Husserliana Bd. XXI, Nijhoff, Den Haag, 1983.

Jaensch, E., « Die Psychologie in Deutschland und die inneren Richtlinien ihrer Forschungsarbeit », Jahrbücher der Philosophie, 3, 1927, 93-168. 
Katz, D., « Die Erscheinungsweisen der Farben und ihre Beeinflussung durch die individuelle Erfahrung », Zeitschrift für Psychologie, Ergänzungsband, 7, 1911, p. 6-31.

Linke, P.F., Die Grundfragen der Wahrnehmungslehre, Munich, Reinhardt, 1918.

Lotze, H., « Seele und Seelenleben », in Wagner, R. (Hrsg.), Handwörterbuch der Physiologie, Bd. IV, n 1, Braunschweig, Vieweg, 1846, p. 172-190.

-, Medizinische Psychologie oder Physiologie der Seele. Leipzig, Weidmann, 1852.

-, Mikrokosmos. Ideen zur Naturgeschichte und Geschichte der Menschheit. Versuch einer Anthropologie, Bd. I. Leipzig, Hirzel, 1856.

-, « Mittheilung an C. Stumpf in Betreff der Lehre von den Localzeichen », in C. Stumpf, Über den psychologischen Ursprung der Raumvorstellung, Leipzig, Hirzel, 1873, p. 315-324.

-, « De la formation de la notion d'espace », Revue philosophique de la France et de l'étranger, IV, 1877, 345-365.

-, System der Philosophie. Zweiter Teil : Drei Bücher der Metaphysik, Leipzig, 1879.

-, Grundzügen der Psychologie. Diktate aus den Vorlesungen, $2^{\mathrm{e}}$ éd., Leipzig, Hirzel, 1881.

-, Grundzügen der Metaphysik. Diktate aus den Vorlesungen, Leipzig, Hirzel, 1883.

Mach, E., Erkenntnis und Irrtum : Skizzen zur Psychologie der Forschung, Leipzig, Barth, ${ }^{2} 1906$.

Pastore, N., Selective History of Theories of Visual Perception : 1650-1950, New York, Oxford University Press, 1971.

Ribot, T., La psychologie allemande contemporaine, Paris, Alcan, 1879.

Scheerer, E., " Motor Theories of Cognitive Structure : A Historical Overview », in Prinz W., Sanders, A. F. (eds.), Cognition and Motor Processes, Berlin, Springer, 1984, p. 77-98.

Stumpf C., Über den psychologischen Ursprung der Raumvorstellung, Leipzig, S. Hirzel, 1873.

-, « Psychologie und Erkenntnistheorie », Abhandlungen der Königlich Bayerischen Akademie der Wissenschaften, München, Franz, 19, 2, 1891, p. 465-516.

-, «Zum Begriff der Lokalzeichen », Zeitschrift für Psychologie und Physiologie der Sinnesorgane, 4, 1893, p. 70-73.

-, «Antrittsrede », Sitzungsberichte der Königlich-Preußischen Akademie der Wissenschaften, Berlin, Reimer, 1895, p. 735-738.

-, « Zur Einteilung der Wissenschaften », Abhandlungen der KöniglichPreußischen Akademie der Wissenschaften, Philosophish-historische Classe, Berlin, Verlag der Königliche Akademie der Wissenschaften, 1906, 1907, p. 1-94 (tr. fr., De la classification des sciences, in Fisette 2006 : 69-254). 
-, " Die Wiedergeburt der Philosophie », Berlin, Francke, 1907, (tr. fr., La renaissance de la philosophie, in Fisette, 2006 : 115-132).

-, « Zum Gedächnis Lotzes », Kant-Studien, 22, 1917, p. 1-26.

-, « Carl Stumpf », in Schmidt, R. (Hrsg.), Die Philosophie der Gegenwart in Selbstdarstellung. V. Leipzig: F. Meiner, 1924, p. 1-57 (trad. fr., Autobiographie, in Fisette, $2006: 255-307)$.

-, Die Attribute der Gesichtsempfindungen, Berlin, Köninglich Preussischen Akademie der Wissenschaften, 8, 1917.

-, « Erkenntnislehre », Bd. I, Leipzig, J. A. Barth, 1939.

-, « Erkenntnislehre », Bd. II, Leipzig, J. A. Barth, 1940.

Turner, R. M., In the Eye's Mind: Vision and the Helmholtz-Hering Controversy, Princeton, Princeton University Press, 1994.

Woodward, W. R., « From Association to Gestalt. The Fate of Hermann Lotze's Theory of Spatial Perception, 1846-1920 », Isis, 69, 1978, p. 572-582.

Wundt, W., Grundzüge der physiologischen Psychologie, Leipzig, Engelmann, 1874

-, «Sur la théorie des signes locaux », Revue philosophique de la France et de l'étranger, VI, 1878, p. 217-231. 